\title{
THE BINARY ENCOUNTER PEAK IN RADIATIVE IONIZATION
}

\author{
D. H. JAKUBASSA-AMUNDSEN \\ Mathematics Institute, University of Munich, \\ Theresienstr. 39, 80333 Munich, Germany \\ e-mail: dj@mathematik.uni-muenchen.de
}

\begin{abstract}
It is shown that the radiative electron capture to continuum in energetic collisions with fast heavy projectiles also leads to a binary-encounter type peak in the high-energy electron spectrum. In contrast to the nonradiative target ionization this peak results from a higher-order process, involving near-elastic scattering, and its shape is different from the target Compton profile. Numerical results within the impulse approximation are presented for $30 \mathrm{MeV} / \mathrm{amu} \mathrm{Kr}^{36+}+\mathrm{H}$ collisions, and the dependence of the peak on collision velocity, target binding and electron emission angle is discussed.
\end{abstract}

With the feasibility of the coincident detection of forward electrons and simultaneously emitted photons during relativistic ion-atom collisions the interest in radiative processes has been revived. The photon is able to carry away the excess energy without notably changing the momentum balance. Therefore radiative processes are, in contrast to the corresponding nonradiative processes, predominantly induced by distant collisions. This leads to a much weaker decrease of the cross section with increasing collision velocity $v$, thereby compensating for the smallness of the radiative coupling constant $e^{2}$ provided $v$ is sufficiently high. In a recent pilot experiment the cusp from radiative capture to the projectile continuum (also termed radiative ionization, RI) could be identified in both the electron and photon spectra from $90 \mathrm{MeV} / \mathrm{amu} \mathrm{U}^{88+}+\mathrm{N}_{2}$ collisions ( $v=56.24$ a.u.) [1]; although even for these collision parameters the RI cusp intensity is still an 
order of magnitude below that for the nonradiative capture to continuum [2]. While the RI cusp corresponds to the short-wavelength limit of the (frame-transformed) electron-projectile nucleus bremsstrahlung $[3,4]$, the binary encounter peak investigated here relates to the longwavelength (LW) bremsstrahlung limit. This LW limit is of particular interest because there the radiative and the nonradiative scattering processes can no longer be distinguished physically. In fact the singly differential cross sections for these two processes become nearly proportional to each other [5, p.136], the divergence of the bremsstrahlung cross section for vanishing photon energy $\omega$ being compensated by the radiative corrections to the scattering of an electron from the projectile Coulomb field [3, §V.25,VI.33], [5, p.191]. However, for RI this $\omega=0$ divergence does not occur because of the finite binding energy of the target electron under consideration, as will be shown below.

It is well known from the nonrelativistic first-order Born theory $[6$, p.19] that the binary encounter (BE) peak from nonradiative target ionization is shaped by the target Compton profile $J_{i}$ [7]. The doubly differential cross section for the ejection of a fast electron with energy $E_{f}$ and momentum $\mathbf{k}_{f}$ into the solid angle $d \Omega_{f}$ by a bare projectile of charge $Z_{P}$ is given by (in atomic units, $\hbar=m=e=1$ ),

$$
\frac{d^{2} \sigma^{B 1}}{d E_{f} d \Omega_{f}}=\frac{4 k_{f} Z_{P}^{2}}{v} \int \frac{d \mathbf{q}}{\left|\mathbf{q}-\mathbf{k}_{f}^{\prime}\right|^{4}}\left|\varphi_{i}^{T}(\mathbf{q}+\mathbf{v})\right|^{2} \delta\left(E_{f}^{\prime}-E_{i}^{T}+\mathbf{q} \mathbf{v}+\frac{v^{2}}{2}\right)
$$

where the final electronic state is represented by a plane wave, $\varphi_{i}^{T}$ is the initial state in momentum space, $-E_{i}^{T}$ the binding energy, and the projectile-frame quantities $\mathbf{k}_{f}^{\prime}=\mathbf{k}_{f}-\mathbf{v}$ and $E_{f}^{\prime}=k_{f}^{\prime 2} / 2$ have been introduced. When the collision velocity is sufficiently high $\left(Z_{T} / v \ll 1\right.$ with $Z_{T}$ the target nuclear charge) the target electron behaves like a quasifree particle. Then the factor $\left|\mathbf{q}-\mathbf{k}_{f}^{\prime}\right|^{-4}$ is of the order of $v^{-4}$ and can be taken outside the integral at $q_{\perp}=0$ where $\varphi_{i}^{T}$ is peaked (for $s$ states). Here, $\mathbf{q}=\left(\mathbf{q}_{\perp}, q_{z}\right)$ and the $z$-axis is taken along $\mathbf{v}$. This peaking approximation renders (1) proportional to $J_{i}\left(q_{z}+v\right)$, where $q_{z}$ is determined by the $\delta$-function. The choice $q_{z}=-v$ (where $J_{i}$ has its maximum) leads to the BE peak position $E_{f} \approx 2 v^{2} \cos ^{2} \vartheta_{f}+2 E_{i}^{T}$ where $\vartheta_{f}$ is the polar angle of $\mathbf{k}_{f}$. This corresponds, if $E_{i}^{T}$ is neglected, to the maximum energy which can be transferred by the projectile to an electron at rest in a binary collision. 
If $Z_{P} \gg Z_{T}$ (the case of present interest) target ionization is more readily described in terms of electron capture to the projectile continuum [8]. In the respective theory the higher-order scattering contributions, although important near the cusp, are negligibly small for the binary encounter electrons [9]. Thus (1) remains a valid description of the BE peak irrespective of $Z_{P}$.

Let us now turn to radiative ionization. When $Z_{P} \gg Z_{T}$ RI can be described within the impulse approximation. The fourfold differential cross section for electron capture into the projectile continuum with the simultaneous emission of a photon of energy $\omega$ into the solid angle $d \Omega$ is given by $[10]$

$$
\begin{gathered}
\frac{d^{4} \sigma^{R I}}{d E_{f} d \Omega_{f} d \omega d \Omega}=\frac{(2 \pi)^{4} k_{f} \omega^{2}}{c^{5} v} \sum_{\lambda} \int d \mathbf{q}\left|\left\langle\psi_{f}^{P}\left|\mathbf{A}_{\lambda} \boldsymbol{\nabla}\right| \psi_{q}^{P}\right\rangle\right|^{2}\left|\varphi_{i}^{T}(\mathbf{q}+\mathbf{v})\right|^{2} \\
\cdot \delta\left(E_{f}^{\prime}-E_{i}^{T}+\omega+\mathbf{q} \mathbf{v}+\frac{v^{2}}{2}\right)
\end{gathered}
$$

where $\mathbf{A}_{\lambda} \boldsymbol{\nabla}$ is the radiation field and $\lambda$ counts the two directions of the photon polarization. Also, $\psi_{q}^{P}$ and $\psi_{f}^{P}$ are projectile continuum eigenstates to momentum $\mathbf{q}$ and $\mathbf{k}_{f}^{\prime}$, respectively.

In order to describe the RI binary encounter peak we have to look more closely at the bremsstrahlung scattering matrix element $\left\langle\psi_{f}^{P}\left|\mathbf{A}_{\lambda} \boldsymbol{\nabla}\right| \psi_{q}^{P}\right\rangle$ which occurs in (2). According to Nordsieck's formula [11] it exists in closed form if hydrogenic wavefunctions are used (see e.g. $[9,12])$. The square of its modulus has, in addition to the $\left|\mathbf{q}-\mathbf{k}_{f}^{\prime}\right|^{-4}$ behaviour from the Coulomb field, a strong singularity $\sim \tilde{\gamma}^{-2}$ at $\tilde{\gamma} \equiv$ $\frac{1}{2}\left(q^{2}-k_{f}^{\prime 2}\right)=0$, corresponding to elastic scattering $q=k_{f}^{\prime}$. Let us insert $\mathbf{q}=-\mathbf{v}$ where $\varphi_{i}^{T}$ has its maximum. Then we get $v=k_{f}^{\prime}=\left|\mathbf{k}_{f}-\mathbf{v}\right|$ which leads to the location of the singularity at $k_{f}=2 v \cos \vartheta_{f}$, i.e. $E_{f}=2 v^{2} \cos ^{2} \vartheta_{f}$. This coincides - up to the order of the target binding energy - with the BE peak position from target ionization. On the other hand energy conservation demands $q_{z}=-\frac{1}{v}\left(E_{f}^{\prime}-E_{i}^{T}+\right.$ $\left.\omega+\frac{v^{2}}{2}\right)<0$ and therefore, $q=k_{f}^{\prime}$ cannot occur. In fact, $q=k_{f}^{\prime}$ implies $-q_{z} \leq k_{f}^{\prime}$ and hence

$$
\frac{1}{2}\left(k_{f}^{\prime}-v\right)^{2}-E_{i}^{T}+\omega \leq 0,
$$


which has no solution because the lhs is strictly positive. Thus, due to the finite binding energy, the scattering is always slightly inelastic and the cross section (2) does not diverge. It is, however, peaked at $k_{f}^{\prime}=v$ and $\omega$ close to zero, as follows from (3). Hence we see how the RI binary encounter peak corrresponds to the long-wavelength limit of bremsstrahlung.

We note that the above arguments remain valid when a relativistic prescription [12] is used, apart from the fact that relativistic kinematics induces a shift of the location of the singularity, i.e. of the RI BE peak position which is then given by $E_{f}=\left(2 v^{2} \cos ^{2} \vartheta_{f}+\right.$ $\left.2 E_{i}^{T} v^{2} / c^{2}\right) /\left(1-v^{2} \cos ^{2} \vartheta_{f} / c^{2}\right)$ to lowest order in $E_{i}^{T}$.

The strong variation of the bremsstrahlung scattering matrix element when the lhs of (3) tends to its minimum value prohibits, in (2), the peaking approximation discussed in the context of (1). Therefore the BE peak from RI is not shaped by the Compton profile. Notice that in contrast to target ionization, it is the lack of a first-order term in the bremsstrahlung process which allows the (higher-order) scattering contributions to become visible in the differential RI cross section.

As seen from Fig.1, where the fourfold differential RI cross section for the $\mathrm{Kr}^{36+}+\mathrm{H} \quad(v=33.85$ a.u. $)$ collision system is plotted at a fixed small photon energy $(\omega=0.1 \mathrm{keV})$, the binary encounter peak is narrower than the Compton profile. On the other hand when the lhs of (3) is large, i.e. for higher photon energies, one moves (even for $k_{f}^{\prime}=v$ ) away from the singularity. Then the bremsstrahlung matrix element varies slowly enough to be taken outside the integral at $q_{\perp}=0$ so that the rhs of (2) becomes proportional to $J_{i}$ which thus shapes the peak (see Fig.1). We note that, due to energy conservation, an increase in $\omega$ leads to a decrease of $E_{f}$ (for vanishing target binding one would strictly have $\left.\frac{v^{2}}{2}=E_{f}^{\prime}+\omega\right)$. When $E_{f}$ has decreased to the cusp region $\left(E_{f} \approx \frac{v^{2}}{2}\right.$, corresponding to $E_{f}^{\prime}=0$ at $\left.\vartheta_{f}=0\right)$ the Compton profile is again modified at forward electron emission angles, in this case by the cusp structure [12].

Integration over the photon energy removes the Compton profile background from the electron spectrum. Therefore, whenever $J_{i}$ dominates the spectrum, the resulting triply differential cross section becomes independent of the electronic initial state. At the BE 
peak, however, in addition to the influence of the near-elastic scattering, only part of the Compton profile falls into the integration region (since $\omega<0$ is not permitted). The resulting strong initial-state dependence in the case of a carbon target can be seen in Fig.2 for the $L$ shell an average over the $s$ and $p$ states was used, represented by a hydrogenic wavefunction with Slater-screened charge and experimental binding energy. Such an initial-state dependence is known from the bremsstrahlung ridge which is visible in the singles (electron-integrated) photon spectrum [13] and corresponds to the short-wavelength limit.

A further integration over the photon angles $\theta, \varphi$ leads to the doubly differential cross section, $d^{2} \sigma / d E_{f} d \Omega_{f}$. In this form the RI yield can be directly compared to the yield from the nonradiative process. As a matter of fact the triply differential RI cross section taken at $\theta=$ $90^{\circ}$ (in the vicinity of which the photon emission is highest) provides the same shape for the spectrum (Fig.2). It is this cross section which is accessible to experiment when all photons emitted into a given solid angle are detected in coincidence with the electrons.

In Fig. 3 the entire electron spectrum from RI is displayed, including the soft-electron peak at $E_{f} \rightarrow 0$ as well as the cusp which is visible at electron ejection angles up to $30^{\circ}$. The position of the binary encounter peak shows the $\cos ^{2} \vartheta_{f}$-behaviour, whereas its shape and peak intensity depend only weakly on the electron emission angle. It remains well separated from the soft-electron peak up to $\vartheta_{f} \approx 70^{\circ}$.

Finally we give a more detailed comparison between the radiative and the nonradiative electron emission. While in the cusp region RI starts to dominate the spectrum at weak-relativistic velocities, the nonradiative binary encounter electrons are emitted with a much larger probability at all velocities which are physically of interest (only in the limit $v \rightarrow c$ does the extra logarithmic dependence on the collision energy favour RI, see [5, p.140] for the underlying bremsstrahlung respective Coulomb scattering processes). This is so because both $\mathrm{BE}$ processes take place at distant collisions whereas cusp electrons without photons require close collisions. For example, for $30 \mathrm{MeV} / \mathrm{amu}$ $\mathrm{Kr}^{36+}+\mathrm{H}$ the BE peak maximum from RI (at $\vartheta_{f} \lesssim 30^{\circ}$ ) is a factor of $10^{-3}$ below the one from target ionization. Moreover, the photons 
emitted simultaneously with the BE electrons in the coplanar geometry considered here are completely polarized $(P=1)$. This feature is independent of the target binding and collision velocity, being related to distant collisions. Again there is the sharp contrast to the strong variation of $P$ for those photons emitted with the cusp electrons.

The minimum velocity required for the visibility of the BE peak above the background is considerably higher for RI and is in addition dependent on $Z_{P}$. For our test system $\left(Z_{P}=36\right)$ we need $v \gtrsim 8$ a.u. (in contrast to $v \gtrsim 5$ a.u. for $Z_{P}=1$ and $v \gtrsim 2.5$ a.u. for the nonradiative process). As a consequence the nonradiative process provides, at fixed velocity, a much larger peak to background ratio. In addition there is a marked dependence on $\vartheta_{f}$ for that process, as concerns the BE peak intensity (which strongly increases with $\vartheta_{f}$ ) as well as the peak to background ratio (which decreases with $\vartheta_{f}$ ), in contrast to the RI results shown in Fig.3. For $30 \mathrm{MeV} / \mathrm{amu} \mathrm{Kr}^{36+}+$ $\mathrm{H}$ the peak to background ratio for the nonradiative process decreases from $2.7 \times 10^{5}$ at $\vartheta_{f}=15^{\circ}$ to $1.7 \times 10^{4}$ at $45^{\circ}$ and to only 15 at $70^{\circ}$. The background intensity was taken at its minimum and calculated with a relativistic version of the higher-order scattering theory from [9]. On the other hand the doubly differential RI cross section provides a ratio slightly below 10 for all $\vartheta_{f} \lesssim 45^{\circ}$ and $\sim 2$ at $70^{\circ}$.

In conclusion we have established that the binary encounter peak in radiative ionization is a signature of higher-order scattering involving eigenstates to the strong projectile field in both the intermediate and final channels. The peak is narrower than predicted by the target Compton profile, the more so the higher the collision velocity, and its shape and height depend only weakly on the electron emission angle for $\vartheta_{f} \lesssim 60^{\circ}$. Since the BE electrons from the nonradiative target ionization have a much higher intensity the study of the RI contribution requires the detection of the emitted photons in coincidence with the electrons.

\section{ACKNOWLEDGMENT}

I would like to thank M.W.Lucas for helpful discussions. 


\section{REFERENCES}

[1] M.Nofal et al, Phys. Rev. Lett. 99 (2007) 163201.

[2] M.Nofal, PhD thesis, University of Frankfurt (2007).

[3] W.Heitler, Quantum Theory of Radiation, $3^{\text {rd }}$ edition, Clarendon Press, Oxford, 1954.

[4] R.H.Pratt, Phys. Rev. 120 (1960) 1717.

[5] D.Bjorken and S.D.Drell, Relativistic Quantum Mechanics, BI, Mannheim, 1964.

[6] J.S.Briggs and J.H.Macek, Adv. Atom. Mol. Opt. Phys. 28 (1991) 1.

[7] D.Burch, H.Wieman and W.B.Ingalls, Phys. Rev. Lett 30 (1973) 823.

[8] K.Dettmann, K.G.Harrison and M.W.Lucas, J. Phys. B7 (1974) 269.

[9] D.H.Jakubassa-Amundsen, J. Phys. B16 (1983) 1767.

[10] D.H.Jakubassa and M.Kleber, Z. Phys. A273 (1975) 29.

[11] A.Nordsieck, Phys. Rev. 93 (1954) 785.

[12] D.H.Jakubassa-Amundsen, J. Phys. B36 (2003) 1971.

[13] T.Ludziejewski et al, J. Phys. B31 (1998) 2601. 


\section{Figure Captions}

Fig.1

Fourfold differential RI cross section for $30 \mathrm{MeV} / \mathrm{amu} \mathrm{Kr}^{36+}+\mathrm{H}$ collisions at electron emission angle $\vartheta_{f}=15^{\circ}$ as a function of kinetic energy $E_{f}$. The solid lines are for photon frequencies $\omega=0.1 \mathrm{keV}$ and $5 \mathrm{keV}$. The photon polar angle is $\theta=90^{\circ}$ and electron and photon are ejected into the same halfplane $(\varphi=0)$.

Also shown $(\cdots \cdots)$ is the Compton profile $J_{1 s}\left(\frac{\omega_{\text {peak }}-\omega}{v}\right)=c_{0}\left(\left(\frac{\omega_{\text {peak }}-\omega}{v}\right)^{2}+Z_{T}^{2}\right)^{-3}$ where $c_{0}$ is fitted to the RI peak maximum. The triply differential cross section, i.e. integrated over all frequencies, is shown as $(-\cdot-\cdot-)$ with the right hand scale. The relativistic RI code from [12] is used throughout, where the peak frequency coincides at $\theta=90^{\circ}$ with the nonrelativistic expression $\omega_{\text {peak }}=E_{i}^{T}-E_{f}+\mathbf{v k}_{f}$. The vertical line marks the 'classic' BE peak position $E_{f}=61.69 \mathrm{keV}$.

Fig.2

Triply differential RI cross section for $30 \mathrm{MeV} / \mathrm{amu} \mathrm{Kr}^{36+}$ projectiles colliding with $\mathrm{H}(-\cdot-\cdot-)$ and $\mathrm{C}(---1 s ;-2 s p)$ per electron at $\vartheta_{f}=15^{\circ}$ as a function of $E_{f}$. The photon parameters are $\theta=90^{\circ}, \varphi=$ 0 .

Also shown, on the right hand scale, is the doubly differential RI cross section for $\mathrm{C}(2 s p)$ per electron $(\cdots \cdots)$.

Fig.3

Triply differential RI cross section for $30 \mathrm{MeV} / \mathrm{amu} \mathrm{Kr}^{36+}+\mathrm{H}$ collisions as a function of $E_{f}$. Results are shown for $\vartheta_{f}=15^{\circ}, 30^{\circ}, 45^{\circ}$ and $70^{\circ}$, and the photon parameters are $\theta=90^{\circ}, \varphi=0^{\circ}$. The vertical line marks the cusp position $E_{f}=(\gamma-1) c^{2}=16.34 \mathrm{keV}$ where $\gamma=\left(1-v^{2} / c^{2}\right)^{-\frac{1}{2}}$. 